\title{
Comprehensive Performance Analysis of a VCSEL-based Photonic Reservoir Computer
}

\author{
Julián Bueno, Joshua Robertson, Matěj Hejda, and Antonio Hurtado, Member, IEEE
}

\begin{abstract}
Optical neural networks offer radically new avenues for ultrafast, energy-efficient hardware for machine learning and artificial intelligence. Reservoir Computing (RC), given its high performance and cheap training has attracted considerable attention for photonic neural network implementations, principally based on semiconductor lasers (SLs). Among SLs, Vertical Cavity Surface Emitting Lasers (VCSELs) possess unique attributes, e.g. high speed, low power, rich dynamics, reduced cost, ease to integrate in array architectures, making them valuable candidates for future photonic neural networks. This work provides a comprehensive analysis of a telecom-wavelength GHz-rate VCSEL RC system, revealing the impact of key system parameters on its performance across different processing tasks.
\end{abstract}

Index Terms-Optical computing, Vertical cavity surface emitting lasers, Neural networks.

\section{INTRODUCTION}

$\mathrm{P}$ hotonic implementations of artificial neural networks (ANN) are receiving increasing research interest thanks to the advantages (e.g. high modulation rates, low power consumption, and unique architectures) offered by photonic hardware technologies [1]-[3]. Among those, Reservoir Computing (RC) is attracting growing attention, as it relies on simple and fast learning methods as it only requires the training of the output layer weights, hence substantially reducing computing time and hardware requirements [4]. RC employs a reservoir where the nodes are randomly interconnected along with self-connections. This creates intrinsic memory inside the ANN, an especially helpful feature in complex data prediction tasks [5], [6]. Furthermore, RC systems can be built with incredibly simplified photonic hardware using a single nonlinear element and a delay line [7]. Called a Time Delay Reservoir (TDR), this concept has been highly useful to develop photonic ANNs based on different devices [8]-[10]. Amongst these, Semiconductor Lasers (SLs) have shown to be excellent candidates for photonic TDRs due to their GHz speed and highly complex dynamics, demonstrating successful ultrafast operation across multiple processing tasks [5], [11]. Vertical Cavity Surface Emitting Lasers (VCSELs) are

This work was supported by the Office of Naval Research Global (ONRGNICOP-N62909-18-1-2027), the European Commission (828841ChipAI-H2020-FETOPEN2018-2020), the UK's EPSRC Doctoral Training Partnership (EP/N509760) and the UKRI Turing AI Acceleration Fellowships Programme (Grant EP/V025198/1). particularly attractive for photonic RC systems due to their technological maturity, reduced cost, low energy operation, ease of integration in 2D/3D architectures, unique polarization properties, and for their extraordinary potential bandwidth that could rise the processing rate to $\mathrm{THz}$ [12], [13]. It is only very recently the first reports have emerged outlining enhanced performance in VCSEL-based RC systems [14], [15]. In order to enable future photonic ANN hardware for use in real-world systems, key work is required to determine their computational performance and the conditions for optimal operation.

This work focuses on this significant challenge reporting a comprehensive experimental analysis of the capabilities of a RC ANN system based on a commercially available $1550 \mathrm{~nm}-$ VCSEL and off-the-shelf fibre-optic components. We investigate its computational performance across different processing tasks, its dependence on critical system parameters and determine the conditions for optimal operation. This work thus provides crucial knowledge to inform future developments in VCSEL RC systems for photonic ANN hardware yielding ultrafast and low-energy operation for machine learning and Artificial Intelligence (AI) functionalities and fully compatible with optical communications and data center technologies.

\section{Delay-BAsed Reservoir COMPUTING}

Whereas in a traditional $\mathrm{RC}$ system the reservoir is a randomly connected network of nonlinear nodes, in a TDR the nodes are unidirectionally arranged in a circle. This is achieved by using a delay line of time $\tau$ and a fast modulated input [7]. The nodes in a TDR are virtual, obtained by interpreting the output of the delay system over different time segments of length $\theta$ as different consecutive nodes. The value of $\theta$ is typically chosen smaller than the characteristic time scale of the nonlinearity to ensure the coupling of each node to the previous ones, therefore creating the network connectivity [7]. We choose $\tau$ to be a multiple of $\theta$, therefore, the number of nodes in the network can be calculated as $N=\tau / \theta$. The information to be processed is a sequence of data inputs, each one injected over a time equal to $\tau$ so it can be mapped into $\mathrm{N}$ virtual nodes. In order to create the injected signal $I(t)$, a temporal mask, made of a sequence of $N$ random values uniformly distributed between 0 and 1 (where each value is held over a time equal to $\theta$ as in a piece wise function) is multiplied to each data input to

The authors are with the Institute of Photonics, Dept. Physics, University of Strathclyde, Technology and Innovation Centre, 99 George Street, Glasgow G11RD, United Kingdom (e-mail: julian.bueno@strath.ac.uk; antonio.hurtado@strath.ac.uk). 
create transient responses at every node.

The output layer computes the output of the RC system based on the state of the reservoir. The weights in this layer, referred as output weights, are the only weights in a $\mathrm{RC}$ system that are optimized and calculated digitally offline via a supervised learning algorithm. To calculate the output weights, we first average the detected signal over bins of time width equal to $\theta$ to obtain the state of the individual nodes to build the averaged response signal ( $x^{\text {train }}$ ) matrix. Then, we split the data samples for training and testing, and use the training section of $x^{\text {train }}$ alongside the labels of the data samples $\left(y^{\text {train }}\right)$ vector in Eq. 1 to calculate the output weights $\left(\omega^{\text {out }}\right)$ vector using the MoorePenrose pseudoinversion matrix operation denoted by ${ }^{+}$. This training phase yields the output weights, given by Eq. 1 .

$$
\omega_{\text {out }}=y^{\text {train }}\left(x^{\text {train }}\right)^{+}
$$

Once the output weights are calculated, we evaluate their performance using the testing section of the response signal to calculate the reservoir output. This is compared with the labels of the testing section to calculate the performance metric.

\section{EXPERIMENTAL SETUP}

The experimental setup of the VCSEL-based RC is depicted in Fig. 1. It consists of off-the-shelf fibre-optic telecom components, including a VCSEL emitting at $1551 \mathrm{~nm}$ with a lasing threshold current of $1.75 \mathrm{~mA}$ at $293 \mathrm{~K}$. The VCSEL's spectrum showed two orthogonally polarised modes, the main lasing (parallel-polarised) mode and a subsidiary attenuated (orthogonally-polarised) mode. These were separated by 0.11 $\mathrm{nm}(14.5 \mathrm{GHz})$ and corresponded to the two orthogonal polarizations of the fundamental transverse mode of the device. No polarization switching was observed for any applied bias current above the VCSEL's lasing threshold. The device is subject to delayed optical feedback using an optical circulator. The delay feedback time was measured to be $65.0 \mathrm{~ns}$; hence by injecting one sample every delay time this system currently processes information at a rate of $\sim 15 \mathrm{MHz}$. A Variable Optical Attenuator (VOA1) and a Polarization Controller (PC1) are used to control the intensity and polarization (parallel or orthogonally-polarised) of the optical feedback, respectively. Under minimum feedback attenuation (maximized feedback strength) $16 \%$ of the light intensity was redirected into the VCSEL. A tunable laser provides CW light (with $25 \mathrm{~dB}$ polarization extinction ratio) and the signal $\mathrm{I}(\mathrm{t})$ is encoded by modulating its intensity with a Mach-Zehnder modulator using

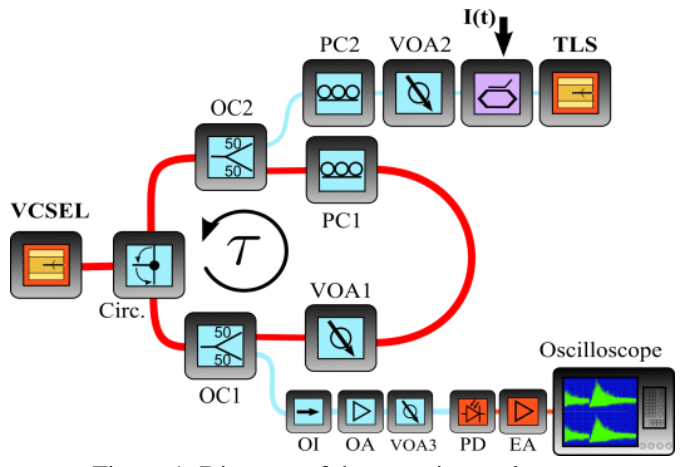

Figure 1. Diagram of the experimental setup. a $5 \mathrm{GHz}$ bandwidth Arbitrary Waveform Generator (AWG) at $5 \mathrm{GSa} / \mathrm{s}$. This modulation rate leads to $\theta=200 \mathrm{ps}$ and to $\mathrm{N}=325$ nodes. A second VOA (VOA2) and PC (PC2) control the intensity and polarization (parallel or orthogonal) of the VCSEL's injected light via an Optical Coupler (OC2). The light sent for detection, extracted using OC1, is amplified optically and electrically, using an Optical Amplifier (OA) and an Electrical Amplifier (EA) respectively, to improve the Signal to Noise Ratio (SNR) before it is recorded with an $8 \mathrm{GHz}$ and 20 $\mathrm{GSa} / \mathrm{s}$ real time oscilloscope.

\section{METhOdS FOR PERFORMANCE EVAlUATION}

To evaluate the performance, we use a prediction task, a classification task, and a memory evaluation. In all methods we used 10000 data inputs randomly divided $80 \%$ for training and $20 \%$ for testing, and cross-validated using 5 folds.

\section{A. The Mackey-Glass prediction task}

In this task the TDR has to predict the next value in a chaotic time series originating from a Mackey-Glass delay equation [4], [16]. We integrated the Mackey-Glass equation with an integration step of 0.17 , and down-sampled to 33 samples per period to obtain the inputs. Performance is assessed calculating the Normalized Mean Square Error (NMSE) with Eq. 2, where $\bar{y}_{i}$ and $y_{i}$ are the predicted and the original time traces, $n_{s}$ is the number of samples, and $\sigma_{y}^{2}$ is the variance of $y_{i}$.

$$
N M S E=\frac{\sum_{i=1}^{N}\left(\bar{y}_{i}-y_{i}\right)^{2}}{n_{s} \sigma_{y}^{2}}
$$

\section{B. The nonlinear channel equalization task}

The goal in this classification task is to recover a 4 amplitude level signal as inputs that has underwent mixing of neighboring information sections, a nonlinear amplitude distortion, and noise [17]. We used a noise strength corresponding to $\mathrm{SNR}=24$ $\mathrm{dB}$. The performance is evaluated by calculating the Symbol Error Rate (SER), as the ratio of symbols incorrectly classified.

\section{Memory evaluation}

To evaluate the memory properties of the VCSEL RC system we calculate its linear memory correlation. To do this, we inject as inputs a stream of pseudo-random numbers $y(\mathrm{k})$ uniformly distributed between 0 and 1 into the reservoir. We train the reservoir to retrieve the number $\bar{y}_{i}(\mathrm{k})$ which was introduced $i$ time steps before. We then calculate the correlation $m_{i}$ for a memory depth $i$ between the original time trace and the reconstructed one as described in Eq. 3. This correlation value measures how well the reservoir can hold and retrieve information that was injected a certain amount of time before.

$$
m_{i}=\operatorname{corr}\left(\bar{y}_{i}(k), y(k-i)\right)
$$

\section{EXPERIMENTAL RESULTS}

\section{A. Performance evaluation on injected power and current}

First, we show the performance dependence for the prediction task. We analyse in Fig. 2 the effect of the average optical injected power $\left(\bar{P}_{i n j}\right)$. We provide results for two different polarization configurations of the optical feedback and 
injection. We define the PAR (ROT) polarization configuration as where the polarization of the optical feedback and injection signals are parallel (orthogonal) with respect to the polarization of the VCSEL's dominant mode. Experimental results are measured for different VCSEL bias currents relative to the measured lasing threshold current in each case $\left(I_{r}=I / I_{t h, f b}\right)$ as reported in Fig. 2. In all cases the optical input power was monotonically increased, and the frequency detuning (defined as the optical frequency difference between the injection and the VCSEL's dominant mode) was set to $\Delta v=-4 \mathrm{GHz}$. For comparison, the grey dashed line included in Fig. 2 indicates the performance of a memoryless linear reservoir in this prediction processing task, calculated by applying the same learning procedure on the injected signal. The results in Fig. 2 show that the NMSE reduces as $\bar{P}_{i n j}$ increases until it reaches a low error level. The minimum NMSE for the ROT and PAR polarisation configurations were equal to $\mathrm{NMSE}=0.012$ with the PAR configuration providing lower overall errors for a broader parameter range. Furthermore, we observe that lower NMSE are attained with bias currents close to the lasing threshold. Also, lower applied bias currents can achieve similar performance than higher current values yet with lower input power requirements.

Fig. 3 shows the performance of our VCSEL RC system on the classification task (under the same conditions as in Fig. 2). Fig. 3 shows that lower error values are obtained for bias currents closer to the device's lasing threshold. In our set parameter space, minima SER values equal to 0.028 and 0.032 were measured for the PAR and ROT configurations; hence revealing a slightly better experimental performance for the PAR configuration for the VCSEL RC system of this work.

\section{B. Performance dependence on frequency detuning}

Fig. 4 shows the impact of the frequency detuning on the performance of the prediction and classification tasks for the two different polarization configurations (with all other parameters fixed). For these experiments we kept $\bar{P}_{\text {inj }}=0.5$ $\mathrm{mW}$ and a bias current to the VCSEL at $1.10 \cdot I_{t h, f b}$. The performance on both tasks reveals a region of low prediction errors achieved around $\Delta v=0 \mathrm{GHz}$, for both the PAR and ROT polarization configurations. VCSELs can undergo injection locking and diverse nonlinear dynamics (e.g. period 1, period 2 , chaos) when subject to external polarised optical injection
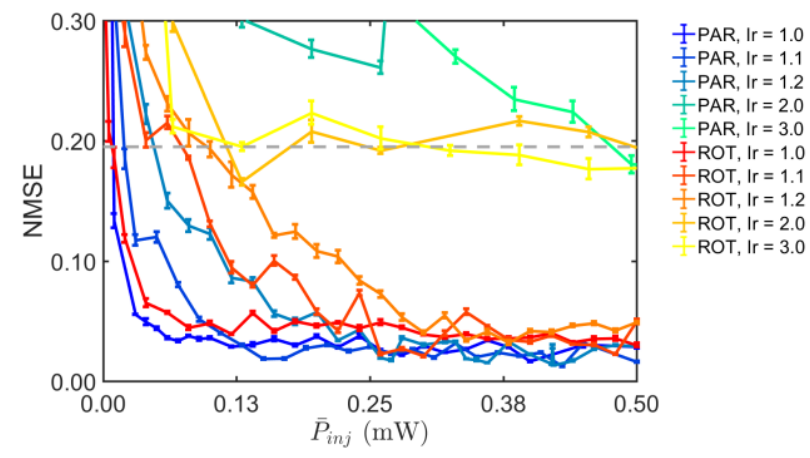

Figure 2. Performance on the prediction task as a function of the average optical injected power for the PAR and ROT configurations and different bias currents (frequency detuning of $\Delta v=-4 \mathrm{GHz}$ ).
[18], [19]. For all cases analysed in this work, the regions where lower error is obtained coincide with the parameter range where the VCSEL is injection-locked to the external signal. In this region, the optical spectrum measured at the systems' output reveals that the VCSEL's emission shifts to the wavelength of the external signal (to which is locked). Errors increase for larger absolute detuning values for which the VCSEL unlocks to the optical input signal. The VCSEL RC system successfully performs both tasks with similar low error values. On the prediction task, the PAR configuration exhibits lower overall error (minimum NMSE of 0.010) than the ROT configuration (minimum NMSE of 0.019). On the classification task, the lowest measured SER values for the PAR (SER=0.036) and ROT $(S E R=0.044)$ polarization configurations are very similar, indicating again that the set polarization does not strongly impact overall system's performance. Interestingly, the performance on either task changes slightly with $\Delta v$ as long as the VCSEL is injection-locked to the external signal.

\section{Memory results}

The memory properties of the VCSEL RC system are shown in Fig. 5. We note the presence of a high memory correlation for depths 6,7 , and 13. Whilst this effect can be seen in the detected signal, this memory does not originate from the inherent dynamics of the VCSEL. We believe this additional memory is introduced by other elements in the setup (e.g. the Mach-Zehnder modulator), or detected from reflected light from the VCSEL. Regardless, we observe the memory correlations have non-zero values and show a continuous profile, characteristic of SLs [5], from memory depth 0 to 8 or 10 with a decaying profile as depth increases. This type of profile can be clearly observed for the PAR configuration in the

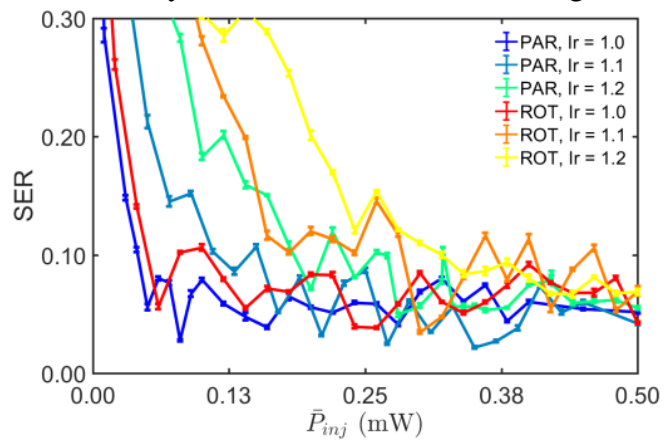

Figure 3. Performance on the classification task as a function of the average optical injected power for the PAR and ROT configurations. Results are presented for different bias currents and a frequency detuning of $\Delta v=-4 \mathrm{GHz}$

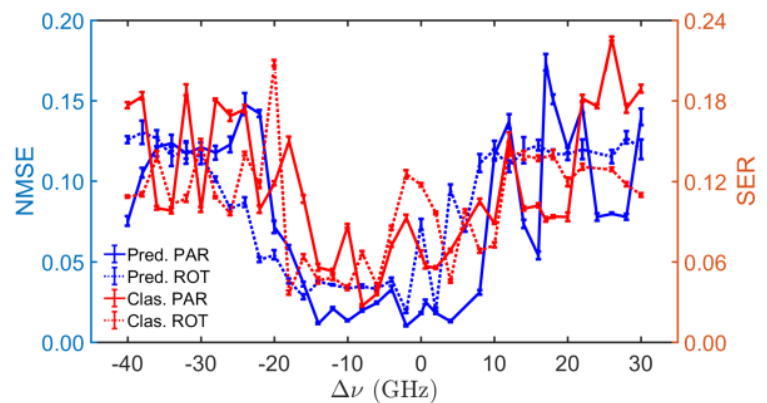

Figure 4. Performance on the prediction (Pred.) and classification (Clas.) tasks for the PAR and ROT configurations as a function of the frequency detuning $(\Delta v)$ for $\bar{P}_{i n j}=0.5 \mathrm{~mW}$ and $I_{r}=1.10$. 
top plot of Fig. 5 for $\bar{P}_{i n j}$ around $0.13 \mathrm{~mW}$. The bottom plot in Fig. 5 also exhibits this profile, albeit weaker, for $\bar{P}_{i n j}$ around $0.25 \mathrm{~mW}$. If the average optical injected power is further increased the memory decreases and collapses. This profile is explained by the VCSEL's injection locking dynamics, where high memory correlation values are found as the VCSEL transitions from an unlocked dynamical state to an injectionlocked state [5], [14], [15]. We hypothesize that as the VCSEL's suppressed orthogonally-polarized mode is noticeably weaker than the dominant parallel polarized mode, using the ROT polarization configuration requires stronger injection locking conditions to obtain similar memory

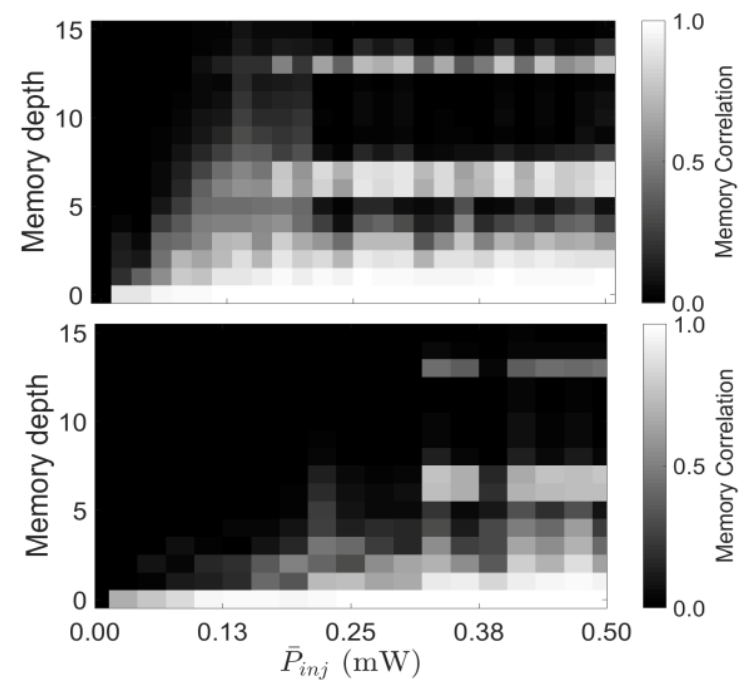

Figure 5. Memory correlation dependence on $\bar{P}_{i n j}$ for the VCSEL-based RC system for $I_{r}=1.10$ and $\Delta v=-4 \mathrm{GHz}$. Results are presented for the PAR (top) and the ROT (bottom) polarization configurations, sharing $\mathrm{x}$-axis. properties.

\section{CONCLUSION}

We report a comprehensive analysis of the performance of a photonic RC system based on an off-the-shelf 1550nm-VCSEL on different processing tasks. Very low error values are achieved for low bias currents and very low optical injected power across a broad parameter range, showing parameter resilience for two different polarization configurations. Our results indicate slightly improved performance for the PAR configuration than for the ROT. We hypothesize that the frequency difference and gain asymmetry of the polarization modes supported by the VCSEL of this work yield experimental conditions favoring the PAR configuration. Tailored VCSELs with engineered optical frequency and gain distributions could improve the ROT configuration's outcome. The lowest error in our system for the studied tasks (NMSE=0.010; SER=0.028) are comparable to those reported in other time-delayed photonic RC systems based on edge-emitting lasers [5] (NMSE=0.019) and VCSELs [15] (SER=0.015). Encouragingly, slightly reduced error $(\mathrm{NMSE}=0.010)$ for the Mackey-Glass prediction task is observed in our VCSEL system when compared to those reported for an edge-emitting laser RC system. It is worth noting that our setup exhibited a SNR between 5 and $10 \mathrm{~dB}$ depending on the injected power, which is lower than in recent works due to the different experimental conditions, and that the system's performance is currently limited by experimental factors such as noise, which create the flat error profiles in our findings. Noise impacts negatively on performance and memory, thus further improving noise conditions is crucial to demonstrate full and new functionalities with VCSEL RC systems. Despite these limitations, the VCSEL RC system of this work, built with off-the-shelf fibre-optic telecom components, delivers excellent processing capabilities and improved performance. This work comprehensively analyzing the effect of key physical parameters on the VCSEL RC system's computational performance and memory operation, opens the door towards future VCSEL-based photonic hardware for machine learning and AI functionalities.

\section{REFERENCES}

[1] J. Feldmann, N. Youngblood, C. D. Wright, H. Bhaskaran, and W. H. P. Pernice, "All-optical spiking neurosynaptic networks with self-learning capabilities," Nature, vol. 569, no. 7755, May 2019.

[2] P. Antonik, N. Marsal, D. Brunner, and D. Rontani, "Human action recognition with a large-scale brain-inspired photonic computer," Nat. Mach. Intell., vol. 1, no. 11, pp. 530-537, Nov. 2019.

[3] J. Moughames et al., "Three-dimensional waveguide interconnects for scalable integration of photonic neural networks," Optica, vol. 7, no. 6, pp. 640-646, Jun. 2020.

[4] H. Jaeger and H. Haas, "Harnessing Nonlinearity: Predicting Chaotic Systems and Saving Energy in Wireless Communication," Science, vol. 304, no. 5667, pp. 78-80, Apr. 2004.

[5] J. Bueno, D. Brunner, M. C. Soriano, and I. Fischer, "Conditions for reservoir computing performance using semiconductor lasers with delayed optical feedback," Opt. Express, vol. 25, no. 3, pp. 2401-2412, Feb. 2017.

[6] A. Argyris, J. Bueno, and I. Fischer, "PAM-4 Transmission at $1550 \mathrm{~nm}$ Using Photonic Reservoir Computing Post-Processing," IEEE Access, vol. 7, pp. 37017-37025, 2019.

[7] L. Appeltant et al., "Information processing using a single dynamical node as complex system," Nat. Commun., vol. 2, no. 1, pp. 1-6, Sep. 2011.

[8] L. Larger et al., "Photonic information processing beyond Turing: an optoelectronic implementation of reservoir computing," Opt. Express, vol. 20, no. 3, pp. 3241-3249, Jan. 2012.

[9] F. Duport, B. Schneider, A. Smerieri, M. Haelterman, and S. Massar, “All-optical reservoir computing," Opt. Express, vol. 20, no. 20, pp. 22783-22795, Sep. 2012.

[10] Q. Vinckier et al., "High performance photonic reservoir computer based on a coherently driven passive cavity," Optica, vol. 2, no. 5, pp. 438-446, Jan. 2015.

[11] A. Argyris et al., "Comparison of Photonic Reservoir Computing Systems for Fiber Transmission Equalization," IEEE J. Sel. Top. Quantum Electron., vol. 26, no. 1, p. 1, 2020.

[12] M. Müller et al., "1550-nm high-speed short-cavity VCSELs," IEEE J. Sel. Top. Quantum Electron., vol. 17, no. 5, pp. 1158-1166, 2011.

[13] M. Lindemann et al., "Ultrafast spin-lasers," Nature, vol. 568, no. 7751, pp. 212-215, 2019.

[14] J. Vatin, D. Rontani, and M. Sciamanna, "Enhanced performance of a reservoir computer using polarization dynamics in VCSELs," Opt. Lett., vol. 43, no. 18, pp. 4497-4500, Sep. 2018.

[15] J. Vatin, D. Rontani, and M. Sciamanna, "Experimental reservoir computing using VCSEL polarization dynamics," Opt. Express, vol. 27, no. 13, pp. 18579-18584, 2019.

[16] M. C. Mackey and L. Glass, "Oscillation and chaos in physiological control systems.," Science, vol. 197, no. 4300. pp. 287-289, 1977.

[17] V. J. Mathews and J. Lee, "Adaptative Algorithms for Bilinear Filtering," in Advanced Signal Processing, 1994, vol. 2296.

[18] A. Hurtado et al., "Nonlinear dynamics induced by parallel and orthogonal optical injection in $1550 \mathrm{~nm}$ Vertical-Cavity SurfaceEmitting Lasers," Opt. Express, vol. 18, no. 9, p. 9423, Apr. 2010.

[19] R. Al-Seyab, K. Schires, N. Ali Khan, A. Hurtado, I. D. Henning, and M. J. Adams, "Dynamics of Polarized Optical Injection in 1550-nm VCSELs: Theory and Experiments," IEEE J. Sel. Top. Quantum Electron., vol. 17, no. 5, pp. 1242-1249, Sep. 2011. 